\title{
Neuromorphic object localization using resistive memories and ultrasonic transducers
}

Filippo Moro ( $\square$ filippomoro.it@gmail.com )

CEA Leti

\section{Emmanuel Hardy}

CEA Leti https://orcid.org/0000-0002-9208-1674

Bruno Fain

CEA Leti

Thomas Dalgaty

CEA Leti

Paul Clemencon

CEA Leti

Alessio De Prá

DPIA, Universitá degli studi di Udine

Niccolò Castellani

CEA, LETI, Minatec Campus, Grenoble

Francois Blard

CEA Leti

Francois Gardien

CEA Leti

Thomas Mesquida

CEA List

Francois Rummens

CEA List

David Esseni

University of Udine

Jérôme Casas

Institut Universitaire de France-IUF

Giacomo Indiveri

University of Zurich and ETH Zurich https://orcid.org/0000-0002-7109-1689

Melika Payvand

University of Zurich and ETH Zurich https://orcid.org/0000-0001-5400-067X

Elisa Vianello

CEA Leti https://orcid.org/0000-0002-8868-9951 
Article

Keywords: sensory-processing, object localization, piezoelectric micromachined ultrasound transducer Posted Date: October 6th, 2021

DOl: https://doi.org/10.21203/rs.3.rs-951481/v1

License: (c) (i) This work is licensed under a Creative Commons Attribution 4.0 International License. Read Full License

Version of Record: A version of this preprint was published at Nature Communications on June 18th, 2022. See the published version at https://doi.org/10.1038/s41467-022-31157-y. 


\title{
Neuromorphic object localization using resistive memories and ultrasonic transducers
}

\author{
Filippo Moro ${ }^{1, *}$, Emmanuel Hardy ${ }^{1}$, Bruno Fain ${ }^{1}$, Thomas Dalgaty ${ }^{1}$, Paul Clémençon ${ }^{1,4}$, \\ Alessio De Prà ${ }^{1,3}$, Niccolò Castellani ${ }^{1}$, François Blard ${ }^{1}$, François Gardien ${ }^{1}$, Thomas \\ Mesquida $^{2}$, François Rummens ${ }^{2}$, David Esseni ${ }^{3}$, Jérôme Casas ${ }^{4}$, Giacomo Indiveri ${ }^{5}$, \\ Melika Payvand ${ }^{5}$, and Elisa Vianello, ${ }^{1,}$
}

\author{
${ }^{1}$ CEA, LETI, Université Grenoble Alpes, Grenoble, France \\ ${ }^{2}$ CEA, LIST, Université Grenoble Alpes, Grenoble, France \\ ${ }^{3}$ DPIA, Università degli Studi di Udine, Udine, Italy \\ ${ }^{4}$ Insect Biology Research Institute, Université de Tours, Tours, France \\ ${ }^{5}$ Institute for neuroinformatics, University of Zurich and ETH Zurich, Switzerland \\ *filippo.moro@cea.fr, elisa.vianello@cea.fr
}

\begin{abstract}
Real-world sensory-processing applications require compact, low-latency, and low-power computing systems. Enabled by their in-memory event-driven computing abilities, hybrid memristive-CMOS neuromorphic architectures provide an ideal hardware substrate for such tasks. To demonstrate the full potential of such systems, we propose and experimentally demonstrate an end-to-end sensory processing solution for a real-world object localization application. Drawing inspiration from the barn owl's neuroanatomy, we developed a bio-mimetic, event-driven object localization system that couples state-of-the-art piezoelectric micromachined ultrasound transducer (pMUT) sensors to a neuromorphic resistive memories-based computational map. We present measurement results from the fabricated system comprising resistive memories-based coincidence detectors, delay line circuits, and a full-custom pMUT sensor. We use these experimental results to calibrate our system-level simulations. These simulations are then used to estimate the angular resolution and energy efficiency of the object localization model. The results reveal the potential of our approach, evaluated in orders of magnitude greater energy efficiency than a microcontroller performing the same task.
\end{abstract}

\section{Introduction}

We are entering an era of pervasive computing, where an exponentially increasing number of devices and systems are being deployed to assist us in our daily lives. These systems are expected to operate continuously, dissipating the lowest possible amount of energy, while learning to interpret the data they capture from several sensors in real-time, and produce a binary output as the outcome of a classification or recognition task. One of the most important steps required to reach this objective is to extract useful and compact information from noisy and often incomplete sensory data ${ }^{1}$. Conventional engineering approaches typically sample the sensed signals at constant and high rates, thus generating huge amounts of data, even in the absence of useful input stimuli. Moreover, these approaches use sophisticated digital signal processing techniques to pre-process the input (often noisy) data. Conversely, biology provides alternative solutions for processing noisy sensory data, using energy-efficient, asynchronous, event (spike)-driven methods ${ }^{2,3}$. Neuromorphic computing draws inspiration from biological systems to reduce the computational cost in terms of energy and memory requirements, relative to conventional signal processing techniques ${ }^{4-6}$. Innovative general-purpose brain-inspired systems that implement spiking neural networks (TrueNorth ${ }^{7}$, BrainScaleS ${ }^{8}$, DYNAP$\mathrm{SE}^{9}$, Loihi ${ }^{10}$, Spinnaker ${ }^{11}$ ) have recently been demonstrated. These processors offer low-power and low-latency solutions for implementing machine learning tasks, and for modeling cortical circuits. To take full advantage of their energy efficiency, these neuromorphic processors should be connected directly to event-driven sensors ${ }^{12,13}$. However, only few sensory devices exist today that directly provide event-driven data. Prominent examples are the Dynamic Vision Sensor (DVS) used for vision applications such as tracking and motion detection ${ }^{14-17}$, the silicon cochlea ${ }^{18}$ and Neuromorphic Auditory Sensor (NAS) ${ }^{19}$, used for processing auditory signals, the olfactory sensor ${ }^{20}$, and multiple examples of touch sensors used for texture recognition ${ }^{21,22}$.

In this article, we present a newly developed event-driven auditory processing system applied to object localization. Here, for the first time, we describe an end-to-end system for object localization that is obtained by coupling the state-ofthe-art piezoelectric micro-machined ultrasound transducers (pMUTs) to a neuromorphic resistive memory (RRAM)-based computational map. In-memory computing architectures employing RRAMs are a promising solution to reduce energy 

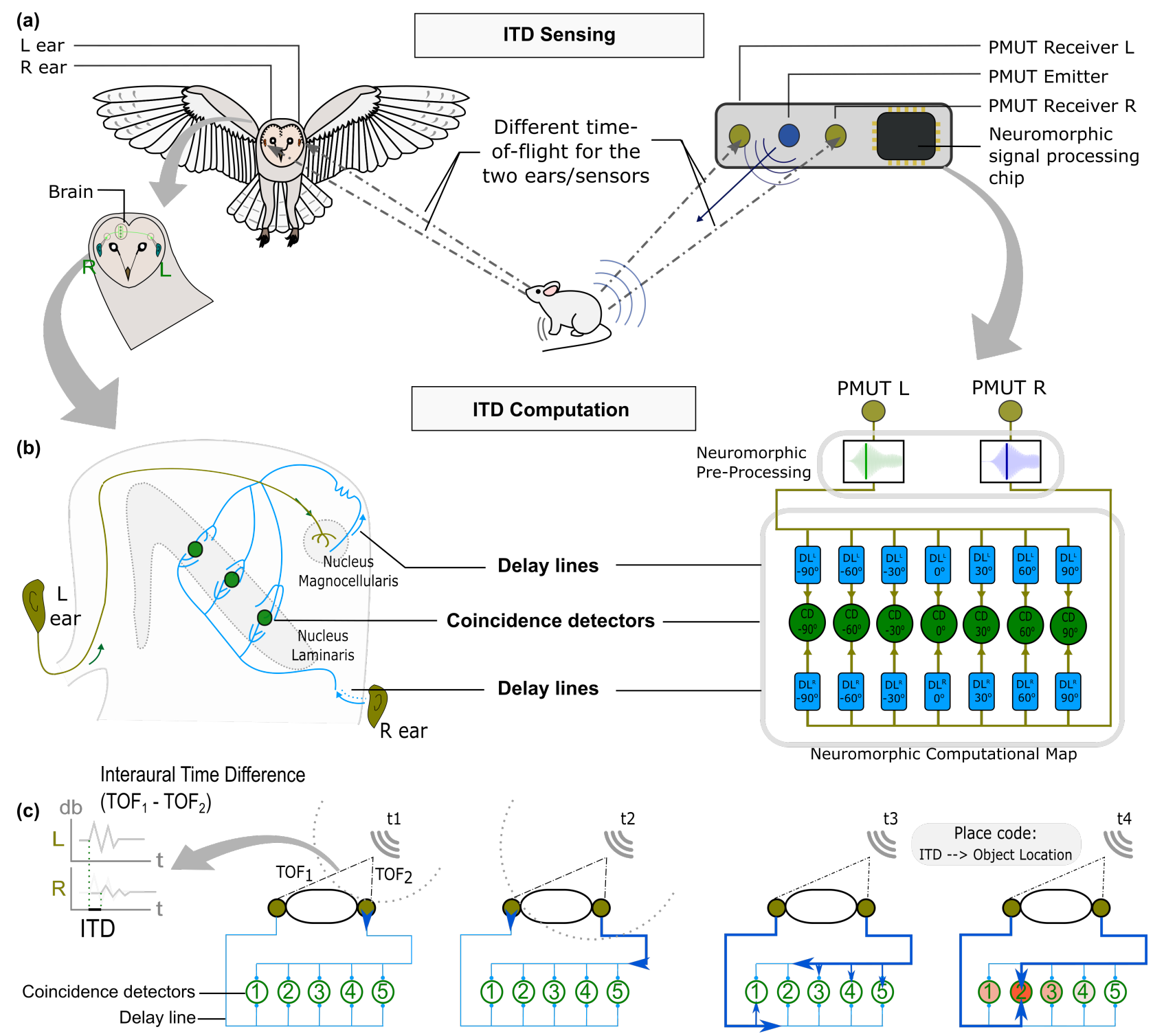

Figure 1. Object localization system in barn owls and the proposed bio-mimetic technology. (a) The barn owl receives a sound wave from a target, a moving prey in this case. The Time-of-Flight (ToF) of the soundwave at each ear is different (unless the prey is exactly in front of the owl). The dash-dotted lines represent the path of the sound wave towards the barn owl's ears. Based on the difference in the two soundwave path lengths and the corresponding Interaural Time Difference (ITD), it is possible to locate the prey precisely in the horizontal plane (left). In our system the pMUT emitter (in dark blue) produces a sound wave that bounces off the targeted object. The reflected ultra-sound wave is sensed by 2 pMUT receivers (in light-green) and processed by the neuromorphic processor (right). (b) The ITD computation model (Jeffress model) describes how sounds reaching the barn owl's ears are first encoded into phase-locked spike trains in the Nucleus Magnocellularis (NM), and then processed using a grid of geometrically arranged coincidence detector neurons in the Nucleus Laminaris (NL) (left). Illustration of the neural ITD computational map combining delay lines and coincidence detector neurons that can be implemented using RRAM-based neuromorphic circuits to model the owl's biological sensing system (right). (c) Diagram of the basic Jeffress mechanism, where the two ears receive a sound stimulus at different moments due to a difference in the ToF and send axons to detectors from opposite ends. The axons are afferent to an array of coincident detector neurons (CDs), each of which is selective to highly temporally correlated inputs. As a result, only the CDs whose inputs arrive with the smallest time difference (the ITD is exactly compensated) will be maximally excited. The CDs will then encode the angular position of the target. 
consumption $^{23-29}$. Their inherent non-volatility - not requiring active power consumption to store or refresh the information - matches the asynchronous event-driven nature of neuromorphic computation perfectly, resulting in virtually no power consumption when the system is idle. Piezoelectric micromachined ultrasound transducers (pMUTs) are low-cost, miniaturized silicon-based ultrasound sensors able to act as emitters and receivers ${ }^{30-34}$. To process the signals captured by the embedded sensors, we have taken inspiration from the neuroanatomy of the barn owl ${ }^{35-37}$. The barn owl Tyto alba is known for its exceptional night hunting capabilities made possible by a very efficient auditory localization system. To calculate the position of a prey, the Barn owl's localization system encodes the Time-of-Flight (ToF) of the sound wave coming from the prey when it reaches each of the owl's ears or sound receptors. Given the distance between the ears, the difference between the two ToF measurements (Interaural Time Difference, ITD) makes it possible to compute the azimuthal location of the target analytically. Although biological systems are not adapted to solve algebraic equations, they perform localization tasks very efficiently. The barn owl's nervous system makes use of an array of Coincidence Detector (CD) neurons ${ }^{35}$ (i.e. neurons able to detect temporal correlations between spikes propagating down converging excitatory terminals) ${ }^{38,39}$ organized into a computational map to solve the localization task.

Previous studies have shown that complementary metal-oxide-semiconductor (CMOS)-based neuromorphic hardware inspired by the inferior colliculus ('auditory cortex') of barn owl constitute an efficient way to compute the position from the ITD ${ }^{13,40-42}$. However, the potential of a full neuromorphic system that couples auditory signals to the neuromorphic computational map has not yet been proven. The main challenge is the intrinsic variability of analog CMOS circuits, affecting the coincidence detection precision. In this work we propose to exploit the ability of RRAMs to change their conductance value in a non-volatile manner to counteract the variability in analog circuits.

We implemented an experimental system consisting of a single emitting pMUT membrane working at $111.9 \mathrm{kHz}$, two reception pMUT membranes (sensors) that emulate the barn owl's ears, and a neuromorphic computational map fabricated by co-integrating a $130 \mathrm{~nm}$ CMOS processor with hafnium dioxide RRAM devices. We experimentally characterized the pMUT sensory system and the RRAM-based ITD computational map to validate our localization system and to estimate its resolution. We compared our approach to a microcontroller performing the same localization task using either conventional beamforming or neuromorphic techniques. We find that the proposed neuromorphic system achieves a reduction in power consumption of five and four orders of magnitude respectively, compared to the two microcontroller-based solutions cited above.

\section{Results}

Biological background. One of the most striking examples of precise and efficient object localization systems can be found in barn owls $35,37,43$. At dusk and dawn, barn owls (Tyto Alba) actively search for small prey such as voles or mice relying mostly on passive listening. These auditory specialists can locate auditory cues incoming from their prey with astonishing accuracy (about $\left.2^{\circ}\right)^{35}$, as shown in Fig. 1a. Barn owls infer the localization of a sound source in the azimuthal (horizontal) plane from the difference between the Time-of-Flight incoming from the source on the two ears (ITD). The ITD computation mechanism has been postulated by Jeffress ${ }^{44,45}$, it relies on neural geometry and requires two key ingredients: axons, neuron's nerve fibers, that act as delay lines, and an array of Coincidence Detector neurons organized into a computational map, as depicted in Fig. 1b. The sound reaches the ears with an azimuth-dependent time delay (Interaural Time Difference, ITD). In each ear, the sound is then converted into a spike pattern. Axons from the left and right ears act as delay lines and converge at $\mathrm{CD}$ neurons. In theory, only one neuron of the array of coincidence neurons will receive simultaneous inputs (where the delay is compensated exactly), and will fire maximally (neighboring cells will fire too, but at a lower rate). This concept is summarized in Fig. 1c: for example, if the sound originates from the right, a coincidence will occur when the input signal from the right ear travels a longer path than from the left ear by an amount compensating the ITD, e.g. at coincidence neuron 2. In other words, each CD responds to a specific ITD (also called Best Delay) because of axonal delays. In this way, the brain transforms temporal information into spatial information. Anatomical evidence has been found for this mechanism ${ }^{37,46}$. There are phase-locked neurons of the Nucleus Magnocellularis who preserve the temporal information of the input sound: as their name indicates, they fire at a specific phase of the signal. The coincidence detector neurons of the Jeffress model can be found in the Nucleus Laminaris. They receive input from neurons of the Nucleus Magnocellularis, whose axons serve as delay lines. The amount of delay provided by delay lines may be explained by axonal lengths, but also by differential myelination patterns, changing the conduction speeds. Inspired by the auditory system of the barn owl, we developed a biomimetic system for object localization. The two ears are represented by the two pMUT receivers. The sound source is a pMUT emitter located in between (Fig. 1a), and the computational map is formed by a grid of RRAM-based CD circuits (Fig. 1b, in green) taking the role of CD neurons, whose inputs are delayed by delay line circuits (in blue) which act as the axons in the biological counterpart.

PMUT sensors for Time-of-Flight measurement. Piezoelectric micromachined ultrasonic transducers are scalable ultrasound sensors that can be integrated with advanced CMOS technology ${ }^{31-33,47}$, and have lower actuating voltage and power consumption than conventional bulk transducers ${ }^{48}$. In our work, the diameter of the membrane is $440 \mu \mathrm{m}$ and the resonance 

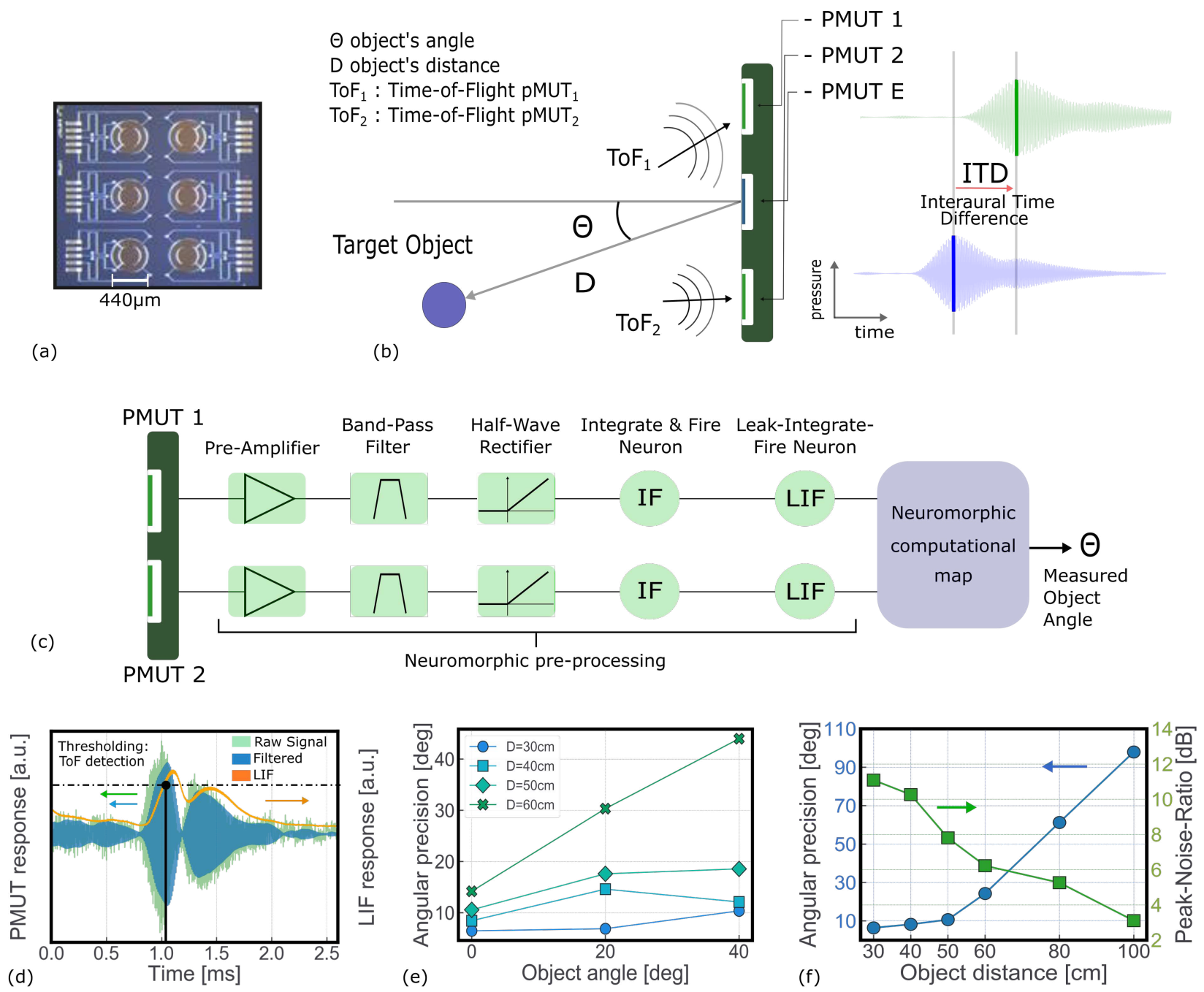

Figure 2. Sensory system assessment. (a) Picture of a pMUT die with six $440 \mu \mathrm{m}$ membranes integrated with a $1.5 \mathrm{~mm}$ pitch. (b) Diagram of the measurement setup. A target object is located at an azimuthal position $\theta$ and distance $\mathrm{D}$. An emitter pMUT produces a wave-form at $111.9 \mathrm{kHz}$ that is reflected by the object and arrives at the 2 receivers pMUTs with different Time-of-Flight (ToF). Such difference, defined as Interaural Time Difference (ITD), encodes the position of the object and can be estimated evaluating the peak of the response in the two receiver sensors. (c) Diagram of the pre-processing steps to convert the raw pMUT signal into a train of spikes (i.e. the input for the neuromorphic computational map). The pMUT sensors and the neuromorphic computational map have been fabricated and tested, while the neuromorphic pre-processing is based on software simulations. (d) Response of the pMUT membrane upon arrival of a signal and conversion to the spike domain. (e)

Experimental angular precision of the localization as a function of the object angle $(\Theta)$ and the distance (D) of the target object. (f) Angular precision (blue line) and corresponding Peak-to-Noise Ratio (green line) as a function of the object distance for $\Theta=0$.

frequency spreads in the range $[110-117] \mathrm{k} \mathrm{Hz}$ (Fig. 2a, see Methods for more details). Over a batch of 10 tested devices, the median quality factor is around $50^{31}$. Combining the information of different membranes is a well-known technique to infer angular information from pMUT devices, using for instance beamforming techniques ${ }^{31,49}$. The potential of this technology for air-borne pulse-echo measurements has been demonstrated using a beamforming strategy on a system composed of a pMUT emitter (composed of 1 membrane) and a pMUT receiver system (made of 5 pMUT membranes arranged in an array with a pitch of $1.5 \mathrm{~mm}$ ), located few centimeters apart from each other ${ }^{31}$. We conducted an experiment locating two pMUT sensors about $10 \mathrm{~cm}$ apart from each other, thus fully taking advantage of the different ToF of sound being sensed by the two receiving membranes. A single pMUT working as an emitter is located in between the receivers. A $12 \mathrm{~cm}$-wide PVC plate located in front of the pMUT devices at a distance D was used as a target (Fig. 2b). The receivers record the sound reflected from the 


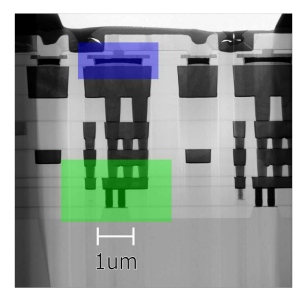

(a)

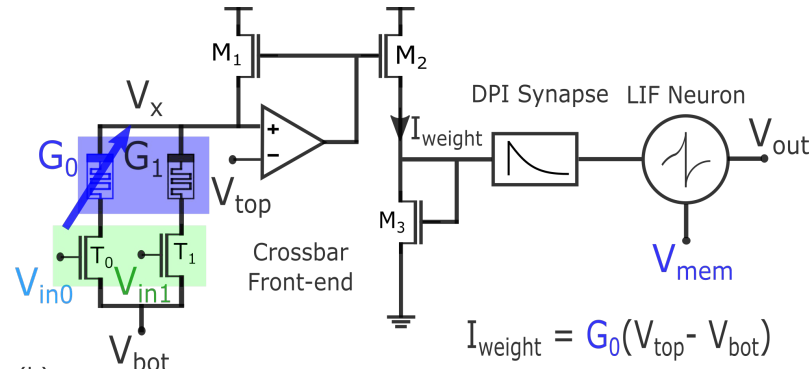

(b)

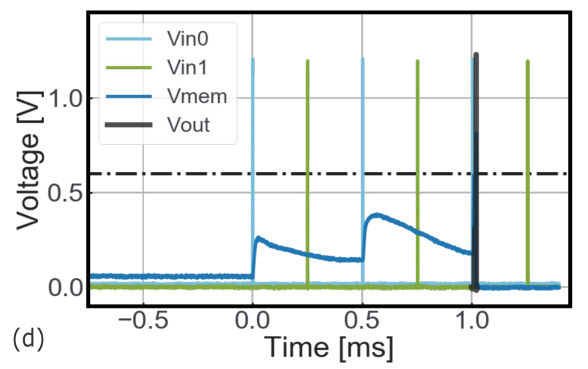

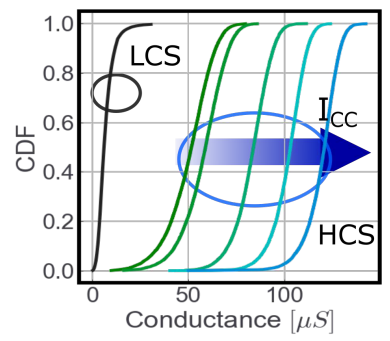

(c)

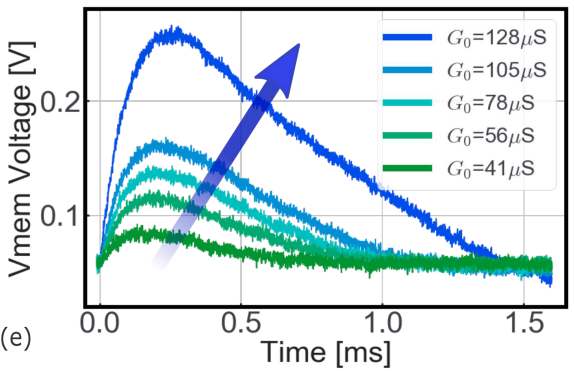

Figure 3. Role of RRAM devices in neuromorphic circuits. (a) Scanning Electron Microscopy (SEM) image of a HfO 2 1T1R RRAM device, in blue, integrated on $130 \mathrm{~nm}$ CMOS technology, with its selector transistor (width of $650 \mathrm{~nm}$ ) in green. (b) Basic building block of the proposed neuromorphic circuit. Inputs voltage pulses (spikes), $V_{i n 0}$ and $V_{i n 1}$, draw a current $I_{w e i g h t}$ proportional to the conductance states, $G_{0}$ and $G_{1}$, of the 1T1R structures. This current is injected into a DPI synapse that excites a LIF neuron. The RRAMs $G_{0}$ and $G_{1}$ are set in the HCS and LCS respectively. (c) Cumulative Density Function of the conductance of a population of $16 \mathrm{~kb}$ RRAM devices, as a function of the compliance current $I_{C C}$, which effectively controls the conductance level. (d) Measurement of the circuit in (a), showing that $G_{1}$ (in LCS) effectively blocks inputs from Vin 1 (green), in fact the output neuron's membrane voltage only responds to the blue input of $\operatorname{Vin}_{0}$. RRAMs efficiently define the connections in the circuit. (e) Measurement of circuit in (b) showing the effect of the conductance value $G_{0}$ on the membrane voltage $V_{\text {mem }}$, following the application of a voltage pulse $V_{i n_{0}}$. The larger the conductance, the stronger the response: the RRAM device thus implements the weight of the input-to-output connection.

object and respond maximally at the Time-of-Flight of the sound wave. The experiment was repeated varying the position of the object, defined by its distance D and its angle $\Theta$. Inspired by ${ }^{50}$, we propose a neuromorphic pre-processing of the pMUT raw signal, as described in Fig. 2c: for each of the two pMUT receivers, the raw signal is band-pass filtered to smooth it out, rectified, and later passed to an Integrate-and-Fire (IF) neuron, which converts the signal into a spike train. This in turn feeds a second Leaky-Integrate-and-Fire (LIF) neuron that produces an output event (spike) in case of overcoming a dynamical threshold (Fig. 2d): the timing of the output spike encodes the detected Time-of-Flight. The threshold of the LIF is calibrated to the pMUT response mitigating the pMUT's device-to-device variability. Thanks to this approach, instead of storing the whole sound wave to memory and process it later, we simply generate a spike at the reception of the sound wave, which constitutes the input of the resistive memory-based computational map. To assess the localization angular precision allowed by the pMUTs and the proposed signal processing technique, we measured the ITD (i.e. the time difference between the spike events generated by the two receivers) when varying the distance and angle of the object. The ITD is then analytically converted into an angle (see Methods) and plotted as a function of the object position: the uncertainty over the measured ITDs grows with both the object's distance and angle (Figs. 2e and f). The main challenge is the noise in the pMUT response. The more distant the object is located, the higher the noise, thus lowering the Peak-to-Noise ratio (Fig. 2f, green line). The decrease in the Peak-to-Noise Ratio (SNR) leads to an increase in the uncertainty over the estimated ITD and consequently on the precision of the localization (Fig. 2f, blue line). For an object located $50 \mathrm{~cm}$ away from the emitter, the system's angular precision is about $10^{\circ}$. This limit, imposed by the sensor's characteristics, can be improved. For example, the emitted signal can be strengthened by coupling several emitters, and/or by using multiple receivers to average the information on the detected Time-of-Flight, and thus lowering the uncertainty. This would extend the range of detection, as demonstrated $\mathrm{in}^{34}$, at the price of an added energy cost.

RRAM-based neuromorphic computational map. Resistive memories store information in their non-volatile conductive states. The basic working principle of this technology is that modifying a material at the atomic level results in changes of its conductance $^{51}$. Here we use an oxide-based resistive memory composed of a $5 \mathrm{~nm}$ hafnium-dioxide layer sandwiched between 
a top and a bottom electrode of titanium and titanium nitride. The conductivity of an RRAM device can be modified by the application of current/voltage waveforms, which create or break a conductive filament composed of Oxygen vacancies between the electrodes. We have co-integrated such devices in a standard $130 \mathrm{~nm}$ CMOS process ${ }^{52}$ to build a fabricated re-configurable neuromorphic circuit implementing coincidence detectors and the delay lines circuits (Fig. 3a). Both the non-volatility and analog nature of the devices perfectly couple with the event-driven nature of the neuromorphic circuits, minimizing power consumption when idle. The basic building block of the proposed circuit is presented in Fig. 3b. It is composed of $N$ parallel one-resistor-one-transistor (1T1R) structures, encoding the synaptic weights, from which a weighted current is extracted and then injected to a common differential pair integrator (DPI) synapse ${ }^{53}$, and finally into a Leaky-Integrate-and-Fire (LIF) neuron ${ }^{54}$ (see Methods for more details). The input spikes are applied at the gates of the 1T1R structures as trains of voltage pulses, with a pulse-width on the order of hundreds of nanoseconds. The resistive memories can be SET into a high conductance state (HCS) by applying an external positive voltage reference on $V_{\text {top }}$ while grounding $V_{\text {bottom }}$, and RESET into a low conductive state (LCS) by applying a positive voltage on $V_{\text {bottom }}$ while grounding $V_{\text {top }}$. The mean value of the HCS can be controlled by limiting the SET programming (compliance) current $\left(I_{\mathrm{CC}}\right)$ via the gate-source voltage of the series transistor (Fig. 3c). The function of RRAMs in the circuit is dual: they route and weigh input pulses.

First, thanks to the two main conductive states (HCS and LCS), the RRAMs can either block or pass the input pulses when they are respectively in the LCS or HCS state. As a result, RRAMs efficiently define the connections in the circuit. This is fundamental to allow the architecture to be re-configurable. In order to prove that, we characterized a fabricated circuit implementation of the circuit block in Fig. 3b. An RRAM corresponding to $G_{0}$ was programmed into the HCS and a second RRAM, $G_{1}$, was programmed in the LCS. Input pulses were applied to both $V_{\text {ino } 0}$ and $V_{\text {in } 1}$. The effect of two input trains of pulses was analyzed in the output neuron, by collecting the membrane voltage and output of the neuron with an oscilloscope. The experiment is successful when only the pulses connected to the neuron by the HCS device $\left(G_{0}\right)$ excite the membrane voltage. This is demonstrated in Fig. 3d, where the blue train of pulses makes the membrane voltage accumulate charge on the membrane capacitor, whereas the green train of spikes leaves the membrane voltage unperturbed.

The second important function of RRAMs is implementing the weight of the connections. By exploiting the analog adjustment of the RRAMs conductance, the input-to-output connection can be appropriately weighted. In a second experiment, the device $G_{0}$ is programmed in different HCS levels and an input pulse is applied to the input $V_{I n 0}$. The input pulse extracts a current from the device $\left(I_{\text {weight }}\right)$ which is proportional to the conductance and the corresponding potential drop $V_{t o p}-V_{b o t}$. This weighted current is then injected into the DPI synapse and output LIF neuron. The membrane voltage of the output neuron is recorded with an oscilloscope and plotted in Fig. 3e. The peak of the neuron membrane voltage responding to a single input pulse is proportional to the conductance of the resistive memory, confirming that RRAMs can be exploited as programmable synaptic weight elements. These two preliminary tests demonstrate that the proposed RRAM-based neuromorphic platform is able to implement the basic elements of the Jeffress basic mechanism, namely the delay line and coincidence detector circuits. The circuital platform is constituted by stacking consecutive blocks, as the one in Fig. 3b, side by side and connecting their gates to common input lines. We designed, fabricated, and tested a neuromorphic platform composed of two output neurons and receiving two inputs (Fig. 4a). The layout of the circuit is shown in Fig. 4b. The upper $2 \times 2$ RRAM matrix allows to route the input pulses to the two output neurons, while the lower $2 \times 2$ array allows the two neurons $\left(N_{0}, N_{1}\right)$ to be recurrently connected. We demonstrate that this platform can assume a delay line configuration and two distinct coincidence detector functionalities, as summarized by the SPICE simulations in Fig. 4c, d, and e.

The Delay Line (Fig. 4c) simply exploits the dynamical behavior of the DPI synapse and LIF neuron to reproduce the input spike from $V_{\text {in } 1}$ to $V_{\text {out } 1}$ with a delay $T_{\text {del }}$. Only the RRAM connecting $V_{\text {in } 1}$ to $V_{\text {out1 }}, G_{3}$, is programmed into the HCS, while the other RRAMs are in the LCS. The $G_{3}$ device is programmed to $140 \mu S$ to ensure that each input spike increases the membrane voltage of the output neuron sufficiently to reach the threshold and to generate a delayed output spike. The delay $T_{\text {del }}$ is defined by both the synapse and neuron time constants. A coincidence detector detects the occurrence of temporally correlated but spatially distributed input signals. A direction insensitive $\mathrm{CD}$ relies on separate inputs converging to a common output neuron (Fig. 4d). The two RRAMs connecting $V_{\text {in } 0}$ and $V_{\text {in } 1}$ to $V_{\text {out } 1}, G_{2}$ and $G_{4}$ respectively, are programmed into the high conductance state. Synchronous arrival of spikes at $V_{\text {in } 0}$ and $V_{\text {in } 1}$ pushes the membrane voltage of the neuron $N_{1}$ over the threshold required to generate an output spike. If the two inputs arrive too far apart in time, the charge on the membrane voltage accumulated by the first input may have time to decay away, preventing the membrane potential of $N_{1}$ to reach the threshold. The $G_{1}$ and $G_{2}$ are programmed to $70 \mu S$, ensuring that a single input spike does not increase the membrane voltage enough to generate an output spike. The direction-sensitive $\mathrm{CD}$ is a circuit sensitive to the spatial order of arrival of impulses: from right to left or vice versa. This is a basic building block in the elementary motion detection network of Drosophila's visual system to compute the direction of motion and detection of collisions ${ }^{55}$. To implement a direction-sensitive $\mathrm{CD}$ the two inputs have to be routed to two different Neurons $\left(N_{0}, N_{1}\right)$ and between those, a directional connection has to be established (Fig. 4e). Upon the arrival of the first input, $N_{0}$ responds by increasing its membrane voltage up to overcoming its threshold and emitting a spike. Thanks to the directional connection in green, this output event in turn excites $N_{1}$. If the $V_{\text {in1 }}$ input event arrives to excite $N_{1}$ 


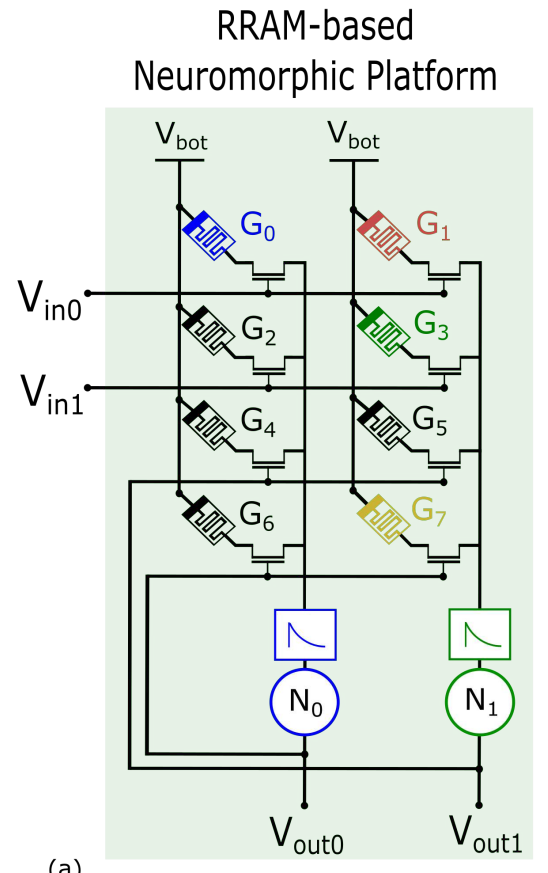

(a)

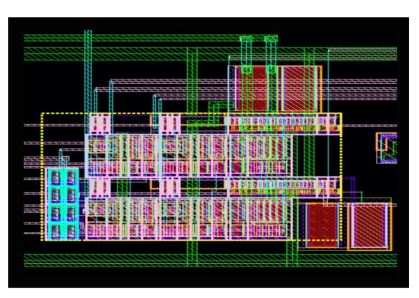

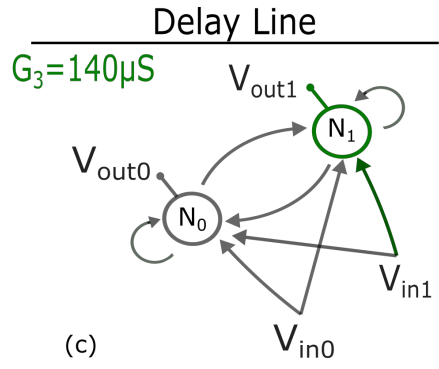
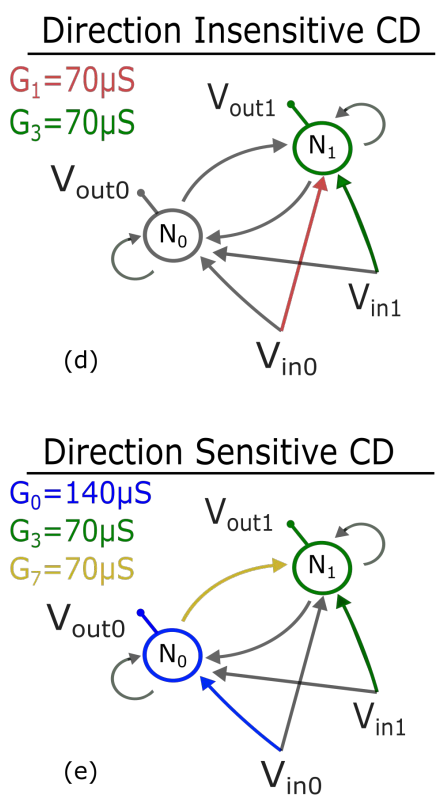
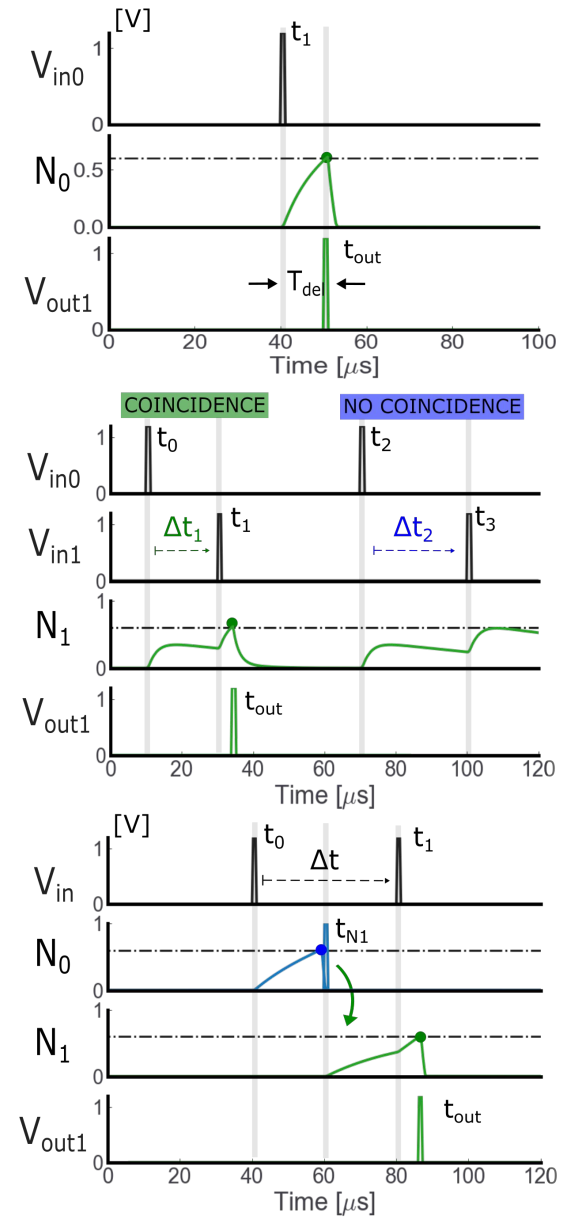

Figure 4. RRAM-based neuromorphic circuital platform. (a) Diagram of the circuit formed by two output neurons, $N_{0}$ and $N_{1}$, receiving two inputs 0 and 1 . The four devices on the top of the array define the synaptic connections from inputs to outputs and the four cells on the bottom define the recurrent connections among neurons. Colored RRAMs represent devices set in the HCS on the diagrams to the right: a device in the HCS allows a connection to be formed and expresses a weight, while a device in the LCS blocks the input pulse and disables the connection to the output. (b) Layout of the circuit in (a), with the 8 RRAMs highlighted in blue. (c) A delay line is formed by simply exploiting the dynamics of a DPI Synapse and a LIF Neuron. The green RRAM is set to high enough conductance to allow the output spike to be elicited following the input spike by a delay $\Delta t$. (d) Diagram of the direction insensitive CD detecting temporally correlated signals. The output neuron $1, N_{1}$, spikes upon arrival of input 0 and input 1 with small delay. (e) Diagram of the direction-sensitive CD, a circuit that detects when input 1 arrives in close proximity and after input 0 . The output of the circuit is represented by neuron $1\left(N_{1}\right)$.

when its membrane voltage is still high, $N_{1}$ will produce an output event, signifying the detection of coincidence between the two inputs. The directional connection allows $N_{1}$ to emit an output only if input 1 arrives after input 0 . The $G_{0}, G_{3}$, and $G_{7}$ are respectively programmed to $140 \mu S, 70 \mu S$, and $70 \mu S$, ensuring that a single input spike at $V_{\text {ino }}$ generates a delayed output spike, while the membrane potential of $N_{1}$ reaches the threshold only upon the synchronous arrival of two input spikes.

Variability in neuromorphic circuits and RRAM-based calibration procedure. Variability is a common source of nonideality in analog neuromorphic systems ${ }^{56-58}$. It results in heterogeneous behaviors among neurons and synapses. Examples of such imperfections include for example 30\% (mean value over standard deviation) of variability on input gain, time constants and refractory period, to name a few (see methods). This issue is more pronounced when several neuron circuits are connected together, as in the case of the direction-sensitive $\mathrm{CD}$, which consists of two neurons. To function properly, the input gain and decay time constants of the two neurons should be as similar as possible. For example, large differences in input gain may result in a neuron responding excessively to an input pulse, while the other being almost insensitive. Fig. 5a shows that randomly selected neurons respond differently to the same input pulse. This neuron variability has an impact on e.g. the 

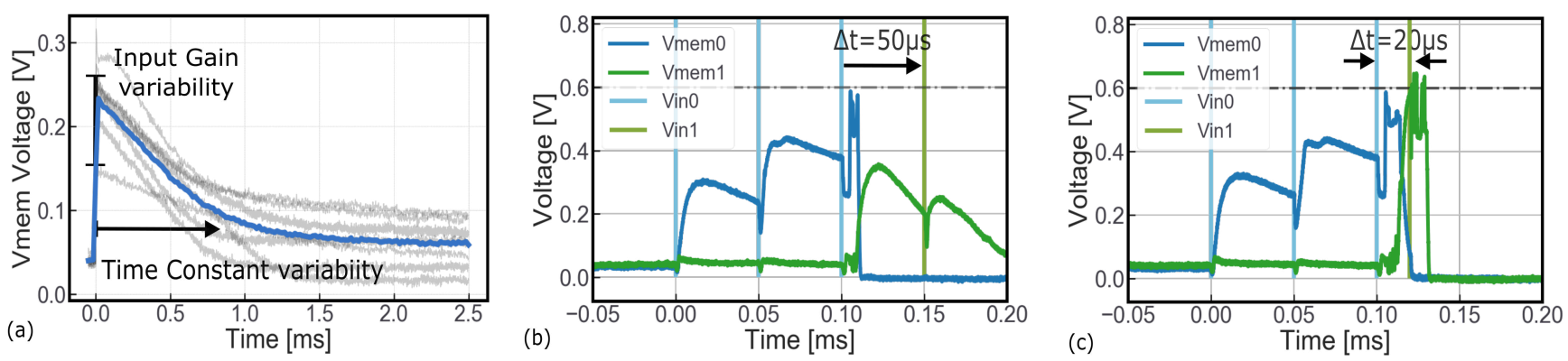

Figure 5. Variability in analog neuromorphic circuits. (a) Response of randomly selected 9 individual neurons to the same input spike. The response varies across the population impacting both the input gain and the time constant. (b) Variability affecting the direction-sensitive CD. Neuron 0 presents a lower input gain than neuron 1, thus requiring 3 input pulses (instead of 1) to produce an output spike. Neuron 1 reaches the threshold with two input events, as expected. If input 1 arrives $\Delta t=50 \mu s$ after neuron 0 has been excited, the CD remains silent because $\Delta t$ is larger than neuron 1's time constant (about $22 \mu s$ ). (c) Decreasing the $\Delta t=20 \mu \mathrm{s}$, makes input 1's spike to arrive when neuron 1's excitation is still high, resulting in the coincidence detection of the two input events.

functionality of the direction-sensitive CD. In the circuit characterized in Fig. 5b and c, neuron 1 presents a much higher input gain than neuron 0 . As a result, neuron 0 requires 3 input pulses (instead of 1) to reach the threshold, while neuron 1 reaches the threshold with two input events, as expected. We propose to exploit the plastic behavior of the resistive memory as a mean of acting on the input gain of neurons and reduce the impact of the neuromorphic circuit variability. As demonstrated in Fig. 4e, the conductance level associated with an RRAM synaptic weight effectively modulates the response of the associated neuron membrane voltage. We adopt an iterative RRAM programming strategy. For a given input, the conductance value of the synaptic weights is re-programmed until the target circuit behavior is obtained (see Method).

The two elements employed in the ITD computational map are the delay lines and direction insensitive CD. Both circuits need to be precisely calibrated to ensure good performance of the object localization system. The delay line has to precisely deliver a delayed-version of the input spike (Fig. 6a), the CD must be activated only when the inputs fall within the target detection range. For the delay line, the synaptic weight of the input connection $\left(G_{3}\right.$ in Fig. $\left.4 \mathrm{a}\right)$ is re-programmed until the target delay is obtained. A tolerance around the target delay is set to stop the procedure: the smaller the tolerance, the harder it is to successfully tune the delay line. Fig. $6 \mathrm{~b}$ shows the result of the calibration procedure for the delay line: as it can be seen the proposed circuit can provide all the delays required in the computational map (from 10 to $300 \mu \mathrm{s}$ ). The maximum number of calibration iterations affects the quality of the calibration procedure: 200 iterations allow the error to be reduced to less than 5\%. One calibration iteration corresponds to a SET/RESET operation of the RRAM cell. The tuning procedure is crucial also to improve the accuracy of the detection of the temporally close events of the CD module. Ten calibration iterations are needed to reach a true positive rate (i.e. rate of events correctly detected as correlated) higher than 95\% (blue line in Fig. 6c). However, the tuning procedure has no effect on false positive events (i.e. rate of events incorrectly detected as correlated). Another technique observed in biological systems which solves the time constraint of rapidly activated pathways is redundancy (i.e. many copies of the same entity are used to fulfill a given function). Taking inspiration from biology ${ }^{59}$, we stacked multiple $\mathrm{CD}$ circuits in each $\mathrm{CD}$ module between two delay lines to reduce the effect of False Positive detection. As shown in Fig. 6c (green lines) stacking $3 \mathrm{CD}$ elements in each $\mathrm{CD}$ module allows reducing the False Positive rate to less than $10^{-2}$.

System assessment We now assess the performance and energy consumption of the object localization system using the results of the electrical characterization of the pMUT sensors, the CD, and the delay line circuits composing the neuromorphic computational map inspired by the Jeffress model (Fig. 1 a). Regarding the neuromorphic computational map, the higher the number of CD modules, the better the angular resolution, but also the higher the system energy (Fig. 7a). A trade-off is reached by comparing the precision of the single components (both pMUT sensors, and neuron and synapse circuits) with that of the whole system. The resolution of delay lines are limited by the time constants of the analog synapses and neurons, which are greater than $10 \mu \mathrm{s}$ in our circuits, corresponding to an angular resolution of $4^{\circ}$ (see Methods). A more advanced CMOS technology node would enable the design of neuron and synapse circuits with lower time constants and consequently higher precision of the delay line element. However, in our system, the precision is limited by the pMUT uncertainty in the estimation of the angular position, that is $10^{\circ}$ (dark-blue horizontal line in Fig. 7a). We fixed the number of CD modules to 40, corresponding to an angular resolution of about $4^{\circ}$, that is the computational map angular precision (light-blue horizontal line in Fig. 7a). At the system level, this results in a $4^{\circ}$ resolution and $10^{\circ}$ precision for an object located in front of the sensory system at a distance of $50 \mathrm{~cm}$. The single bank power consumption for the pre-processing of the pMUT signal is evaluated at $12.3 \mathrm{nW}$, 


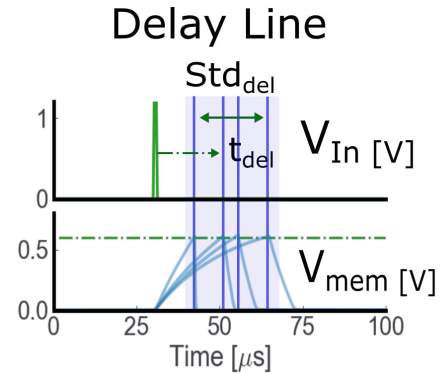

(a)
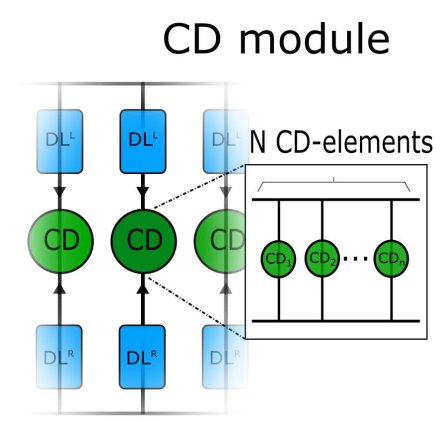

(c)
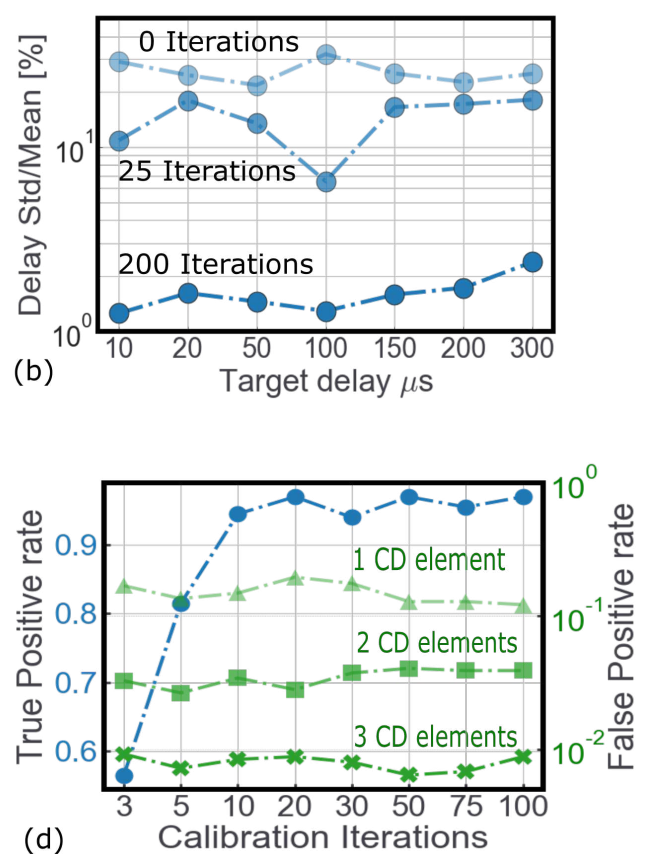

(d)

Figure 6. Performance of the delay line and direction insensitive CD circuits and impact of the RRAM calibration procedure. (a) Impact of the neuron variability on the delay line circuit. (b) The delay line circuit can be scaled up to larger delays, setting the time constant of the corresponding LIF neuron and DPI synapse to larger values. Increasing the iterations of the RRAM calibration procedure enabled us to substantially improve the precision on the target delay: 200 iterations allow the error to be reduce to less than 5\%. One iteration corresponds to a SET/RESET operation on the RRAM cell. (c) Each CD module in the Jeffress Model can be implemented with $N$-parallel CD elements, to be more resilient to system failures. (d) More RRAM calibration iterations allow to improve the True Positive rate (blue line), while the False Positive rate is independent of the number of iterations (green lines). Stacking more CD elements in parallel enabled us to avoid False Coincidence Detection from a CD Module.

according to ${ }^{50}$. Accounting for the $40 \mathrm{CD}$ modules in the computational map, the energy per operation (i.e. energy to localize an object) estimated by SPICE simulations is $21.6 \mathrm{~nJ}$. The neuromorphic system is activated only at the arrival of an input event, i.e. when the sound wave reaches any of of the pMUT receivers and overcomes the detection threshold, and kept idle otherwise. This allows avoiding unnecessary energy consumption when no input signal is present. Considering a rate of localization operations of $100 \mathrm{~Hz}$ and an activation period of $300 \mu s$ per operation (maximum possible ITD), the power consumption of the neuromorphic computational map results being of $61.7 \mathrm{nW}$. Accounting for the neuromorphic pre-processing applied to each of the pMUT receivers brings the total system's power consumption at $86.3 \mathrm{nW}$. To gain a perspective on the energy efficiency of the proposed neuromorphic approach compared to conventional hardware, we benchmark this figure to the energy required for running the same task on a state-of-the-art low-power microcontroller ${ }^{60}$ using either neuromorphic or conventional beamforming techniques. The neuromorphic method accounts for an Analog-Digital-Converter (ADC) stage followed by a Band-Pass filter and an envelope extraction stage (Teager-Kaiser method). Finally, a thresholding operation is performed to extract the ToF. We omit the computation of the ITD based on the ToF and the conversion to the estimated angular position as it happens once per measurement (see Methods). Assuming a sampling frequency of $250 \mathrm{kHz}$ on the two channels (pMUT receivers), 18 operations for the band-pass filter, 3 operations for the envelope extraction and 1 operation for the thresholding per sample, the estimate of the overall power consumption leads to $0.9 \mathrm{~mW}$. The power consumption for the beamforming signal processing solution proposed in $^{31}$, accounting for 5 pMUT receivers and 11 beams equally distributed in the $\left[-50^{\circ},+50^{\circ}\right]$ azimuthal plane is $11.71 \mathrm{~mW}$ (see Method for the details). Based on these estimations, the proposed neuromorphic approach achieves a reduction of five orders of magnitudes in power consumption relative to a microcontroller adopting a classical beamforming technique for an object localization operation. Adopting a neuromorphic signal processing approach on a classical microcontroller reduces the power consumption of about one order of magnitude. The efficiency of the proposed system can be attributed to the combination of the asynchronous, resistive memory-based analog circuits able to perform in-memory computing, along with the absence of analog-to-digital conversion of the sensed signal. 


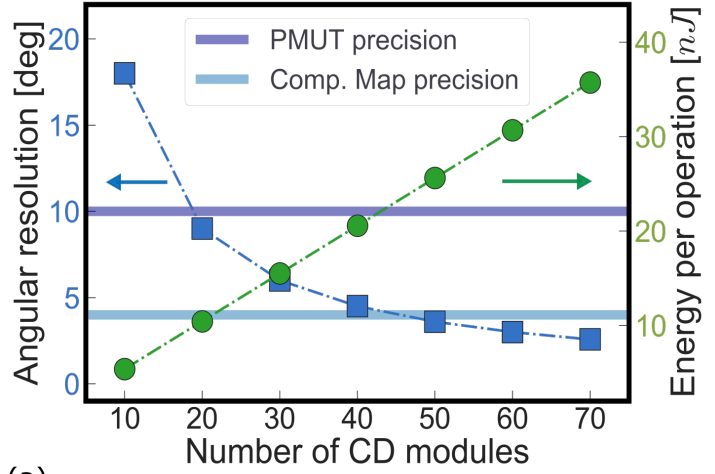

(a)

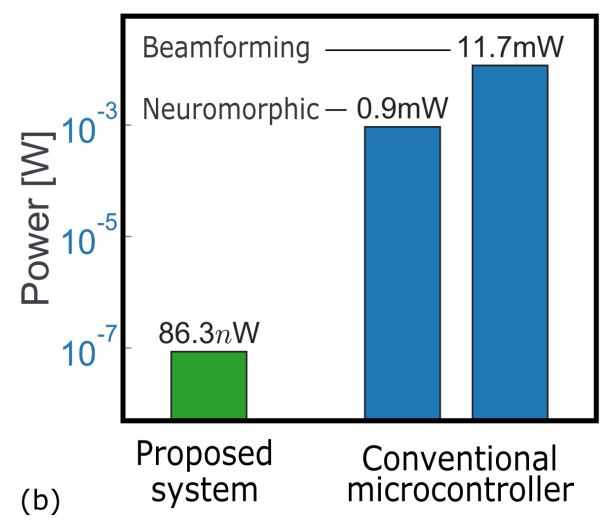

(b)

Figure 7. Power consumption and angular resolution of the presented neuromorphic sensory and signal processing system. (a) Angular resolution (blue) and energy consumption (green) of one localization operation as a function of the number of CD modules. The dark-blue horizontal bar represents the angular precision of the PMUTs while the light-blue horizontal bar represents the angular precision of a the neuromorphic computational map. (b) Power consumption of the proposed system and comparison with the two discussed implementations on a microcontroller.

\section{Conclusion}

To minimize the energy consumption of the object localization system, we envisioned, designed, and implemented an efficient, event-driven RRAM-based neuromorphic circuit that processes signal information produced by embedded sensors to calculate a targeted object's position in real time. Whereas conventional processing techniques would continuously sample the detected signal and crunch calculations to extract the useful information, the proposed neuromorphic solution computes asynchronously as the useful information arrives: this has allowed us to increase the system's energy efficiency by 5 orders of magnitude. Furthermore, we highlight the flexibility of RRAM-based neuromorphic circuits. The capability of RRAMs to change their conductance in a non-volatile manner (plasticity) compensates for the inherent variability of the ultra-low-power analog DPI synapse and neuron circuits. This makes such RRAM-based circuits versatile and powerful. Our goal is not to extract complex features or patterns from a signal, but to locate an object in real-time. Our system is also capable of efficiently compressing the signal and eventually sending it to a subsequent processing stage, if needed, for more complex decision-making. In the context of a localization application, our neuromorphic pre-processing stage can provide information about the position of a target. This information can be used, for example, for motion detection or gesture recognition. We stress the importance of coupling ultra-low-power sensors, such as pMUTs, with ultra-low-power electronics. To do so, the neuromorphic approach has been key, as it led us to the design of novel circuit implementations of biologically-inspired computational methods such as the Jeffress model. In the context of sensor fusion application, our system can be coupled with several different event-based sensors to compute more accurate information. Although owls can perfectly locate their prey in the dark, they have an excellent vision, and prey capture is preceded by combined auditory and visual search ${ }^{61}$. When a specific auditory neuron fires, the owl has the information needed to determine in which direction to start the visual search, focusing its attention on only small subsets of the visual scene. Combining visual sensors (DVS cameras) and the proposed audition sensor (pMUT based) should be explored to develop future autonomous agents.

\section{Methods}

Acoustic measurement setup and pMUT characterization. pMUT sensors are arranged in a printed circuit board, separating the two receivers of about $10 \mathrm{~cm}$, with the emitter between the receivers. In this work, each membrane is a suspended bimorph structure made of two $800 \mathrm{~nm}$-thick piezoelectric AlN layers sandwiched between three $200 \mathrm{~nm}$-thick Molybdenum (Mo) layers, covered by a $200 \mathrm{~nm}$-thick top SiN passivation layer, as reported in ${ }^{62}$. Inner and outer electrodes are patterned on the bottom and the top Mo layers, while the middle Mo electrode is not patterned and used as the ground, resulting in a membrane with four electrodes pairs. In front of the pMUTs, we positioned a rectangular plate, of about $50 \mathrm{~cm}^{2}$, reflecting the emitted sound wave. Both the distance of the plate and the angle with respect to the pMUT plane are controlled utilizing dedicated supports. Tectronix CPX400DP voltage sources bias the three pMUT membranes to tune the resonant frequency to $111.9 \mathrm{kHz}^{31}$, while the emitter is controlled by a Tectronix AFG 3102 pulse generator set close to the resonance frequency $(111.9 \mathrm{kHz})$, and a duty cycle of 0.01 . The currents read at the 4 output ports of each pMUT receiver is converted into a voltage by a dedicated differential current-to-voltage architecture and the resulting signal is digitized by a Spektrum acquisition system.

We characterized the limit of detection by collecting the pMUT signal in different conditions: we moved the reflecting 
plate at different distances $[30,40,50,60,80,100] \mathrm{cm}$ and varied the angle of the pMUT support $\left([0,20,40]^{\circ}\right)$. Fig. S2b shows the relationship between the temporal resolution in detecting Interaural Time Difference (ITD) and the corresponding angular position in degrees.

Design and fabrication of neuromorphic circuits. Two different fabricated RRAM circuits are used in this Article. The first is a $16384(16 \mathrm{k})$ device array (128 x 128 devices) of one transistor/one resistor, 1T1R, structures. The second chip is the neuromorphic platform presented in Fig. 4a. The RRAM cell consists of $\mathrm{HfO}_{2} 5 \mathrm{~nm}$ thick film sandwiched in a TiN/HfO $/ 2 \mathrm{Ti} / \mathrm{TiN}$ stack. The RRAM stack is integrated into the Back End Of Line (BEOL) of a standard 130nm CMOS process. RRAM-based neuromorphic circuits present the challenge of designing a fully analog electronic system in which the RRAM devices coexist with conventional CMOS technology. In particular, the conductive state of the RRAM devices has to be read and utilized as a functional variable of the system. To do so, a circuit that reads a current from a device upon arrival of an input pulse and that uses such current to weight the response of a Differential Pair Integrator (DPI) synapse has been designed, fabricated, and tested. The circuit is shown in Fig. 3a and it represents the basic building block of the neuromorphic platform in Fig. 4a. The input pulses activate the gate of the 1T1R devices, resulting in a current flow through the RRAM proportional to the conductance of the device, $G\left(I_{\text {weight }}=G\left(V_{\text {top }}-V_{x}\right)\right.$ ). The operational amplifier circuit (OPAMP) has a constant DC bias voltage $V_{\text {Top }}$ applied to its inverting input. The negative feedback of the OPAMP will act to ensure that $V_{x}=V_{\text {top }}$, by sourcing an equal current from transistor $M_{1}$. The current extracted from the device, $I_{\text {weight }}$, is injected onto the DPI synapse. Stronger currents will result in greater depolarization, thus the RRAM's conductance effectively implements the synaptic weight. This exponential synaptic current is injected onto the membrane capacitor of a leaky-integrate and fire (LIF) neuron where it integrates as a voltage. If the threshold voltage of the membrane (the switching voltage of an inverter) is overcome, the output section of the neuron is activated, producing an output spike. This pulse feeds back and shunts the neuron membrane capacitor to ground such that it is discharged. The circuit is then complemented by a pulse extender, not shown in Fig. 3a, that reshapes the output pulse of the LIF neuron to the target pulse width. Further multiplexers were integrated on each line in order to be able to apply voltages to the top and bottom electrodes of the RRAM devices.

Circuit measurement setup and RRAM characterization. The electrical tests involved analysing and recording the dynamical behavior of analog circuits as well as programming and reading RRAM devices. Both phases required dedicated instrumentation, all simultaneously connected to the probe card. RRAMs devices in the neuromorphic circuits are accessed from the external instrumentation by means of multiplexars (MUXs). The MUXs decouple the 1T1R cell from the rest of the circuit where they belong, allowing to read and/or program the device. For programming and reading the RRAM devices, a Keithley 4200 SCS machine was used combined with an Arduino microcontroller: the first for precise pulse generation and current reading, the second to fast access a single 1T1R element in the memory array. The first operation is the forming of the RRAM devices. The cells are selected one by one and a positive voltage was applied between the top and bottom electrodes. At the same time, the current is limited to the order of tens of micro-amperes by applying an appropriate gate voltage to the selector transistor. Afterwards, the RRAM cells can be cycled between the Low Conductance State (LCS) and the High Conductance State (HCS) through RESET and SET operations, respectively. SET operations are performed with a positive square voltage pulse of $1 \mu s$ width and $[2.0-2.5] \mathrm{V}$ peak voltage applied to the Top Electrode, and a similarly shaped synchronous pulse with $[0.9-1.3] V$ peak voltage applied to the gate of the selector transistor. Such values allow to modulate the RRAM conductance in the $[20-150] \mu S$ interval. For the RESET, a pulse of $1 \mu s$ width and $3 \mathrm{~V}$ peak is applied to the Bottom Electrode (Bit Line) of the cell while the gate voltage is in the $[2.5-3.0] \mathrm{V}$ range. Inputs and outputs of the analog circuits are dynamical signals. In the case of the input, we have alternated two HP 8110 pulse generators with a Tektronix AFG3011 waveform generator. Input pulses have a width of $1 \mu \mathrm{s}$ and rise/fall edge of $50 \mathrm{~ns}$. This type of pulse is assumed as the stereotypical spiking event in the spike-based analog circuit. Concerning the outputs, a $1 \mathrm{GHz}$ Teledyne LeCroy oscilloscope was utilized to record the output signals. The acquisition speed of the oscilloscope has been proven not to be a limiting factor analysing and collecting data from the circuits.

Variability in neuron and synapse circuits Exploiting analog electronics' dynamics to mimic the behavior of neurons and synapses is an elegant and efficient solution to improve the computation efficiency. The downside of such a computational substrate is that it is subject to variability, from circuit to circuit. We quantified the variability in neuron and synapse circuits (Fig.S1(a,b) in the Supplementary Material). Of all the manifestations of variability, the most impactful at the system level are the ones concerning the time constant and the input gain. The time constant of LIF neurons and DPI synapses is defined by an $R C$ circuit, where the $R$ value is controlled by a bias voltage applied to a transistor's gate ( $V_{l k}$ for neurons, $V_{\text {tau }}$ for synapses), defining the leakage rate. Input gain is defined as the peak voltage reached by the synapse and neuron membrane capacitor stimulated by an input pulse. The input gain is controlled by another biased transistor modulating the input current. Monte Carlo Simulations calibrated on ST Microelectronics $130 \mathrm{~nm}$ process are performed in order to collect some statistics about the input gain and the time constant. The results are plotted in Fig.S1 (Supplementary Material) where input gain and time constant 
are quantified as a function of the bias voltage controlling the leakage rate. Green markers quantify the standard deviation of the time constants with respect to the mean value. Both neuron and synapse circuits are capable of expressing a wide range of time constants, in the $\left[10^{-5}-10^{-2}\right] \mathrm{s}$ range, as shown in Fig.S1 (c,d) (Supplementary Material), while the variability is quantified at $30 \%$ for both the neuron and synapse circuit. The input gain (Fig.S1 (e,d)) (Supplementary Material) variability is around $8 \%$ and 3\% for the neuron and synapse respectively. Such defects are well documented in the literature: in the family of DYNAP chips, different measurements have been performed to evaluate the mismatch across a population of LIF Neurons ${ }^{56}$. Synapses in the Mixed-Signal chip BrainScale have been measured and the mismatch between them analyzed, also proposing a calibration procedure to reduce the variability impact at the system level ${ }^{57}$.

RRAM-based calibration procedure The function of RRAMs in neuromorphic circuit is dual: defining the architecture (routing inputs to outputs) and implementing synaptic weights. The latter property can be exploited to mitigate the problem of variability in analog neuromorphic circuits. We developed a simple calibration procedure that consists in re-programming the RRAM device until the circuit under analysis meets certain requirements. For a given input, the output is monitored and the RRAMs are re-programmed until the target behavior is achieved. The synaptic weights are adapted or calibrated to the requirements of the analog neuromorphic circuit. Focusing on the two basic functionalities of the Neuromorphic Platform, the delay lines and direction insensitive $\mathrm{CD}$, the calibration procedure is summarized in Algorithms [1,2] (Supplementary Material). For the delay line circuit, the target behavior is to deliver the output pulse with a delay $\Delta t$. If the actual delay of the circuit is smaller than the target, the synaptic weight $G_{3}$ has to be decreased (the $G_{3}$ has to be RESET and then SET with a lower compliance current, $I_{c c}$ ). Contrarily, if the actual delay is longer than the target, the conductance of $G_{3}$ has to be reinforced (the $G_{3}$ has to be RESET and then SET with a higher $I_{c c}$ ). This procedure is repeated until the delay produced by the circuit matches the target, with a tolerance set to stop the calibration procedure. For the direction insensitive $\mathrm{CD}$, the calibration procedure involves two RRAM devices, $G_{1}$ and $G_{3}$. The circuit is provided with two inputs, $V_{i n 0}$ and $V_{i n 1}$ delayed by $d t$. The circuit must only respond to delays lower than the coincidence range $\left[0, d t_{C D}\right]$. When an output spike is absent whereas the input spikes are close, both the RRAM devices must be reinforced in order to help the neuron reach the threshold. Conversely, if the circuit responds to delays larger than the target range $d t_{C D}$, the conductances have to be decreased. The procedure is repeated until the correct behavior is obtained. The compliance current can be modulated by the embedded analog circuit presented in ${ }^{63,64}$. Exploiting this embedded circuit, one could perform such procedure periodically to calibrate the system or to re-purpose it for different applications.

Estimation of the power consumption on a microcontroller We estimate the power consumption of a neuromorphic signal processing approach on an off-the-shelf 32-bits microcontroller ${ }^{60}$. In this estimation, we assumed to operate with an identical setup to the one presented in this work, with 1 pMUT emitter and 2 pMUT receivers. The method accounts for a Band-Pass filter followed by an envelope extraction stage (Teager-Kaiser method), and finally a thresholding operation applied to the signal to extract the Time-of-Flight. The computation of the ITD and its conversion to the detected angle are omitted in the estimation. We consider the Band-Pass filter to be implemented with a $4^{\text {th }}$ order Infinite Impulse Response filter, requiring 18 floating-point operations. Envelope extraction makes use of further 3 floating-point operations, and a final operation is due for thresholding. In total, 22 floating-point operations are required for pre-processing the signal. The signal sent is a short pulse of a $111.9 \mathrm{kHz}$ sine wave, produced every $10 \mathrm{~ms}$, resulting in a $100 \mathrm{~Hz}$ localization operation frequency. We adopt a $250 \mathrm{kHz}$ sample rate to respect the Nyquist theorem and a $6 \mathrm{~ms}$ window per measurement to capture a range of $1 \mathrm{~m}$. Note that $6 \mathrm{~ms}$ is the Time-of-Flight for an object located at $1 \mathrm{~m}$ distance. This gives a power consumption of $180 \mu \mathrm{W}$ for the analog-to-digital conversion of 0.5 MSPS. The pre-processing of the signal accounts for 6.60 MIPS (instructions per second), resulting in $0.75 \mathrm{~mW}$. Adding those two contributions makes for a total of $0.93 \mathrm{~mW}$ of power consumption. Note that we omit the derivation of the ITD from the ToFs and the conversion to the detected angle, thus underestimating the power consumption in the microcontroller. This gives further value to the energy efficiency of the proposed system. As a further term of comparison, we estimate the power consumption of a classical beamforming approach, presented $\mathrm{in}^{31,49}$, when embedded on the same microcontroller ${ }^{60}$ under a $1.8 \mathrm{~V}$ supply voltage. Five evenly spaced pMUT membranes are used to provide data for the beamforming. About the processing itself, the beamforming technique used is the Delay-and-Sum. It simply consists in applying a delay to the channels corresponding to the expected time difference of arrival between one channel and a reference channel. If the signals are in phase, once time-shifted, the sum of these signals will exhibit high energy. If they are not in phase, destructive interference will limit the energy of their sum. $\mathrm{In}^{31}$, a $2 \mathrm{MHz}$ sample rate is chosen to time-shift the data by an integer number of samples. A more frugal approach consists in keeping a coarser $250 \mathrm{kHz}$ sample rate and using Finite-Impulse-Response (FIR) filters to synthesize fractional delays. We will consider that the beamforming algorithm complexity is dominated by the time-shifting because of the convolution of each channel with a 16 taps FIR filter for each direction. To calculate the number of MIPS required for this operation, we consider using a $6 \mathrm{~ms}$ window per measurement to capture a 1-meter range, 5 channels, 11 beamforming directions $\left(+/-50^{\circ}\right.$ range with a $10^{\circ}$ step). Already 75 measurements per second push the microcontroller to its maximum of 100 MIPS. According to ${ }^{60}$, this results in a power consumption of $11.26 \mathrm{~mW}$, which gives a total power consumption of $11.71 \mathrm{~mW}$ 
when adding the on-chip ADC contribution.

\section{Data availability}

All the measured data are available upon request.

\section{Code availability}

All software programs used in the presentation of the Article are available upon request.

\section{Acknowledgements}

We acknowledge funding support from the H2020 MeM-Scales project (871371) as well as the French ANR via Carnot funding. In addition, we thank J.-F. Nodin, F. Andrieu, S. Bonnetier, T. Hirtzlin (CEA-Leti), D. Querlioz (Université Paris-Saclay, CNRS) and A. Valentian (CEA-List) for discussing various aspects of the article.

\section{Author contributions}

F.M, T.D, M.P and E.V. developed the idea of processing pMUT sensory input with neuromorphic RRAM-based circuits. F.M, E.H, B.F, F.B, F.G, T.M and F.R contributed to the measurement and analysis of the pMUT sensory system. P.C and J.C provided insights into the anatomy of the barn owl's auditory system and proposed ideas to implement the biological concepts in circuits. T.D and M.P designed and laid out the RRAM-based circuits, and F.M and A.D.P characterized them. E.V. directed the work. All authors contributed to writing the paper.

\section{References}

1. Indiveri, G. \& Sandamirskaya, Y. The importance of space and time for signal processing in neuromorphic agents: The challenge of developing low-power, autonomous agents that interact with the environment. IEEE Signal Process. Mag. 36, 16-28, DOI: 10.1109/MSP.2019.2928376 (2019).

2. Thorpe, S. J. Spike arrival times: A highly efficient coding scheme for neural networks. Parallel Process. Neural Syst. Comput. 91-94 (1990).

3. Levy, W. B. \& Calvert, V. G. Communication consumes 35 times more energy than computation in the human cortex, but both costs are needed to predict synapse number. Proc. Natl. Acad. Sci. 118, DOI: 10.1073/pnas.2008173118 (2021). https://www.pnas.org/content/118/18/e2008173118.full.pdf.

4. Dalgaty, T., Vianello, E., De Salvo, B. \& Casas, J. Insect-inspired neuromorphic computing. Curr. Opin. Insect Sci. 30, 5966, DOI: https://doi.org/10.1016/j.cois.2018.09.006 (2018). Neuroscience Insect bio-inspired micro and nanotechnologies.

5. Roy, K., Jaiswal, A. \& Panda, P. Towards spike-based machine intelligence with neuromorphic computing. Nature 575, 607-617, DOI: https://doi.org/10.1038/s41586-019-1677-2 (2019).

6. Indiveri, G. \& Liu, S.-C. Memory and information processing in neuromorphic systems. Proc. IEEE 103, 1379-1397, DOI: 10.1109/JPROC.2015.2444094 (2015).

7. Akopyan, F. et al. Truenorth: Design and tool flow of a $65 \mathrm{mw} 1$ million neuron programmable neurosynaptic chip. IEEE Transactions on Comput. Des. Integr. Circuits Syst. 34, 1537-1557, DOI: 10.1109/TCAD.2015.2474396 (2015).

8. Schemmel, J. et al. Live demonstration: A scaled-down version of the brainscales wafer-scale neuromorphic system. In 2012 IEEE International Symposium on Circuits and Systems (ISCAS), 702-702, DOI: 10.1109/ISCAS.2012.6272131 (2012).

9. Moradi, S., Qiao, N., Stefanini, F. \& Indiveri, G. A scalable multicore architecture with heterogeneous memory structures for dynamic neuromorphic asynchronous processors (dynaps). IEEE Transactions on Biomed. Circuits Syst. 12, 106-122, DOI: 10.1109/TBCAS.2017.2759700 (2018).

10. Davies, M. et al. Loihi: A neuromorphic manycore processor with on-chip learning. IEEE Micro 38, 82-99, DOI: 10.1109/MM.2018.112130359 (2018).

11. Furber, S. B., Galluppi, F., Temple, S. \& Plana, L. A. The spinnaker project. Proc. IEEE 102, 652-665, DOI: 10.1109/ JPROC.2014.2304638 (2014).

12. Liu, S.-C. \& Delbruck, T. Neuromorphic sensory systems. Curr. Opin. Neurobiol. 20, 288-295, DOI: https://doi.org/10. 1016/j.conb.2010.03.007 (2010). Sensory systems. 
13. Schoepe, T. et al. Neuromorphic sensory integration for combining sound source localization and collision avoidance. In 2019 IEEE Biomedical Circuits and Systems Conference (BioCAS), 1-4, DOI: 10.1109/BIOCAS.2019.8919202 (2019).

14. Risi, N., Aimar, A., Donati, E., Solinas, S. \& Indiveri, G. A spike-based neuromorphic architecture of stereo vision. Front. Neurorobotics 14, 93, DOI: 10.3389/fnbot.2020.568283 (2020).

15. Osswald, M., Ieng, S.-H., Benosman, R. \& Indiveri, G. A spiking neural network model of 3Dperception for event-based neuromorphic stereo vision systems. Sci. Reports 7, 1-11, DOI: 10.1038/srep40703 (2017).

16. Dalgaty, T. et al. Insect-inspired elementary motion detection embracing resistive memory and spiking neural networks. In Biomimetic and Biohybrid Systems, vol. 10928, 115-128, DOI: https://doi.org/10.1007/978-3-319-95972-6_13 (2018).

17. D’Angelo, G. et al. Event-based eccentric motion detection exploiting time difference encoding. Front. Neurosci. 14, 451, DOI: 10.3389/fnins.2020.00451 (2020).

18. V. Chan, S. L. \& van Schaik, A. Aer ear: A matched silicon cochlea pair with address event representation interface. IEEE Transactions on Circuits Syst. I: Regul. Pap. 54, 48-59, DOI: 10.1109/TCSI.2006.887979 (2007).

19. Jiménez-Fernández, A. et al. A binaural neuromorphic auditory sensor for fpga: A spike signal processing approach. IEEE Transactions on Neural Networks Learn. Syst. 28, 804-818, DOI: 10.1109/TNNLS.2016.2583223 (2017).

20. Imam, N. \& Cleland, T. Rapid online learning and robust recall in a neuromorphic olfactory circuit. Nat Mach Intell 2, 181-191, DOI: 10.1038/s42256-020-0159-4 (2020).

21. Bartolozzi, C., Natale, L., Nori, F. \& Metta, G. Robots with a sense of touch. Nat. Mater. 15, 921-925, DOI: https: //doi.org/10.1038/nmat4731 (2016).

22. Mastella, M. \& Chicca, E. A hardware-friendly neuromorphic spiking neural network for frequency detection and fine texture decoding. In 2021 IEEE International Symposium on Circuits and Systems (ISCAS), 1-5, DOI: 10.1109/ ISCAS51556.2021.9401377 (2021).

23. Dalgaty, T. et al. In situ learning using intrinsic memristor variability via markov chain monte carlo sampling. Nat. Electron. 4, 151-161, DOI: https://doi.org/10.1038/s41928-020-00523-3 (2021).

24. Ambrogio, S. et al. Equivalent-accuracy accelerated neural-network training using analogue memory. Nature 558, 60-67, DOI: https://doi.org/10.1038/s41586-018-0180-5 (2018).

25. Chicca, E. \& Indiveri, G. A recipe for creating ideal hybrid memristive-CMOS neuromorphic processing systems. Appl. Phys. Lett. 116, 120501, DOI: 10.1063/1.5142089 (2020).

26. Xia, Q. \& Yang, J. Memristive crossbar arrays for brain-inspired computing. Nat. Material 18, 309-323, DOI: https: //doi.org/10.1038/s41563-019-0291-x (2019).

27. Ielmini, D. \& Wong, H.-S. In-memory computing with resistive switching devices. Nat. Electron. 1, 333-343, DOI: https://doi.org/10.1038/s41928-018-0092-2 (2018).

28. Prezioso, M. et al. Training and operation of an integrated neuromorphic network based on metal-oxide memristors. Nature 521, 61-64, DOI: https://doi.org/10.1038/s41928-018-0092-2 (2015).

29. Esmanhotto, E. et al. High-density $3 \mathrm{~d}$ monolithically integrated multiple 1t1r multi-level-cell for neural networks. In 2020 IEEE International Electron Devices Meeting (IEDM), 36.5.1-36.5.4, DOI: 10.1109/IEDM13553.2020.9372019 (2020).

30. Przybyla, R. J. et al. In-air rangefinding with an aln piezoelectric micromachined ultrasound transducer. IEEE Sensors J. 11, 2690-2697, DOI: 10.1109/JSEN.2011.2157490 (2011).

31. Fain, B., Blard, F., Bastien, J.-C., Gardien, F. \& Frassati, F. Beamforming with aln-based bimorph piezoelectric micromachined ultrasonic transducers. In 2021 Smart Systems Integration (SSI), 1-4, DOI: 10.1109/SSI52265.2021. 9467016 (2021).

32. Muralt, P. et al. Piezoelectric micromachined ultrasonic transducers based on pzt thin films. IEEE Transactions on Ultrason. Ferroelectr. Freq. Control. 52, 2276-2288, DOI: 10.1109/TUFFC.2005.1563270 (2005).

33. Herrera, B., Pop, F., Cassella, C. \& Rinaldi, M. Aln pmut-based ultrasonic power transfer links for implantable electronics. In 2019 20th International Conference on Solid-State Sensors, Actuators and Microsystems Eurosensors XXXIII (TRANSDUCERS EUROSENSORS XXXIII), 861-864, DOI: 10.1109/TRANSDUCERS.2019.8808320 (2019).

34. Zhen, Z., Shinya, Y. \& Shuji, T. Epitaxial pmnn-pzt/si mems ultrasonic rangefinder with $2 \mathrm{~m}$ range at $1 \mathrm{v}$ drive. Sensors Actuators A: Phys. 266, 352-360, DOI: https://doi.org/10.1016/j.sna.2017.09.058 (2017).

35. Knudsen, E. I. \& Konishi, M. Emechanisms of sound localization in the barn owl (tyto alba). J. Comp. Physiol 133, 13-21 (1979). 
36. Takahashi, T., Bala, A. \& Spitzer, M. The synthesis and use of the owl's auditory space map. Biol. Cybern. 89, 378-387, DOI: https://doi.org/10.1007/s00422-003-0443-5 (2003).

37. Carr, C. \& Konishi, M. A circuit for detection of interaural time differences in the brain stem of the barn owl. J. Neurosci. 10, 3227-3246, DOI: https://doi.org/10.1523/JNEUROSCI.10-10-03227.1990 (1990).

38. Bender, V. A., J., B. K., J., B. D. \& Feldman, D. E. Two coincidence detectors for spike timing-dependent plasticity in somatosensory cortex. J. Neurosci. 26, 4166-4177, DOI: https://doi.org/10.1523/JNEUROSCI.0176-06.2006 (2006).

39. Caillard, O., Ben-Ari, Y. \& Gaiarsa, J.-L. Long-term potentiation of gabaergic synaptic transmission in neonatal rat hippocampus. J. Phisiology 518, 109-119, DOI: https://doi.org/10.1111/j.1469-7793.1999.0109r.x (1999).

40. Mead, C., Arreguit, X. \& Lazzaro, J. Analog vlsi model of binaural hearing. IEEE 2, 230-236, DOI: https://doi.org/10. 1109/72.80333 (1991).

41. Sarbashis, D., Akhil, D. \& Saptarshi, D. A biomimetic 2d transistor for audiomorphic computing. Nat. Commun. 10, 3450, DOI: https://doi.org/10.1038/s41467-019-11381-9 (2019).

42. Pfeil, T., Scherzer, A.-C., Schemmel, J. \& Meier, K. Neuromorphic learning towards nano second precision. In The 2013 International Joint Conference on Neural Networks (IJCNN), 1-5, DOI: 10.1109/IJCNN.2013.6706828 (2013).

43. Funabiki, K., Ashida, G. \& Konishi, M. Computation of interaural time difference in the owl's coincidence detector neurons. J. Neurosci. 31, 15245-15256, DOI: https://doi.org/10.1523/JNEUROSCI.2127-11.2011 (2011).

44. Jeffress, L. A. A place theory of sound localization. J. Comp. Physiol. Psychol. 41, 35-39, DOI: https://doi.org/10.1111/j. 1469-7793.1999.0109r.x (1948).

45. S., H. N. \& David, M. Optimal neural population coding of an auditory spatial cue. Nature 430, 682-686, DOI: https://doi.org/10.1038/nature02768 (2004).

46. Joris, P. X., Smith, P. H. \& Yin, T. C. T. Coincidence detection in the auditory system: 50 years after jeffress. Neuron 21, 1235-1238 (1998).

47. Rozen, O. et al. Monolithic mems-cmos ultrasonic rangefinder based on dual-electrode pmuts. In 2016 IEEE 29th International Conference on Micro Electro Mechanical Systems (MEMS), 115-118, DOI: 10.1109/MEMSYS.2016.7421571 (2016).

48. Jiang, X., Luo, G.-L., Wang, Q. \& Horsley, D. A. Improving pmut transmit performance via sub-micron thickness scaling. In 2018 IEEE International Ultrasonics Symposium (IUS), 1-9, DOI: 10.1109/ULTSYM.2018.8580158 (2018).

49. Przybyla, R. et al. In-air ultrasonic rangefinding and angle estimation using an array of aln micromachined transducers. 50-53, DOI: 10.31438/trf.hh2012.14 (2012).

50. Yang, M. et al. Nanowatt acoustic inference sensing exploiting nonlinear analog feature extraction. IEEE J. Solid-State Circuits 1-1, DOI: 10.1109/JSSC.2021.3076344 (2021).

51. Vianello, E. et al. Resistive memories for ultra-low-power embedded computing design. In 2014 IEEE International Electron Devices Meeting, 6.3.1-6.3.4, DOI: 10.1109/IEDM.2014.7046995 (2014).

52. Grossi, A. et al. Experimental investigation of 4-kb rram arrays programming conditions suitable for tcam. IEEE Transactions on Very Large Scale Integration (VLSI) Syst. 26, 2599-2607, DOI: 10.1109/TVLSI.2018.2805470 (2018).

53. Bartolozzi, C. \& Indiveri, G. Synaptic dynamics in analog vlsi. Neural Comput. 19, 2581-2603, DOI: 10.1162/neco.2007. 19.10.2581 (2007).

54. Indiveri, G. et al. Neuromorphic silicon neuron circuits. Front. Neurosci. 5, 73, DOI: https://doi.org/10.3389/fnins.2011. 0007 (2011).

55. Haag, J., Arenz, A., Serbe, E., Gabbiani, F. \& Borst, A. Complementary mechanisms create direction selectivity in the fly. eLife 5, 1-15, DOI: https://doi.org/10.7554/eLife.17421 (2016).

56. Neftci, E. \& Indiveri, G. A device mismatch compensation method for vlsi neural networks. In 2010 Biomedical Circuits and Systems Conference (BioCAS), 262-265, DOI: 10.1109/BIOCAS.2010.5709621 (2010).

57. Aamir, S. A. et al. An accelerated lif neuronal network array for a large-scale mixed-signal neuromorphic architecture. IEEE Transactions on Circuits Syst. I: Regul. Pap. 65, 4299-4312, DOI: 10.1109/TCSI.2018.2840718 (2018).

58. Payvand, M., Nair, M. V., Müller, L. K. \& Indiveri, G. A neuromorphic systems approach to in-memory computing with non-ideal memristive devices: from mitigation to exploitation. Faraday Discuss 213, 487-510, DOI: 10.1039/C8FD00114F (2019). 
59. Faisal, A., Selen, L. \& Wolpert, D. Noise in the nervous system. Nat. Rev. Neurosci. 9, 292-303, DOI: https://doi.org/10. 1038/nrn2258 (2008).

60. ST-Micro. Ultra-low-power arm cortex-m4 32-bit mcu+fpu, 100dmips, $128 \mathrm{~kb}$ flash, $40 \mathrm{~kb}$ sram, analog, aes.

61. D'Souza, P., Liu, S.-C. \& Hahnloser, R. H. R. Perceptron learning rule derived from spike-frequency adaptation and spike-time-dependent plasticity. Proc. Natl. Acad. Sci. 107, 4722-4727, DOI: 10.1073/pnas.0909394107 (2010). https://www.pnas.org/content/107/10/4722.full.pdf.

62. Jung, J. et al. Wafer-level experimental study of residual stress in aln-based bimorph piezoelectric micromachined ultrasonic transducer. Eng. Res. Express 2, DOI: 10.1088/2631-8695/abc140 (2020).

63. Payvand, M., Demirag, Y., Dalgaty, T., Vianello, E. \& Indiveri, G. Analog weight updates with compliance current modulation of binary rerams for on-chip learning. In 2020 IEEE International Symposium on Circuits and Systems (ISCAS), 1-5, DOI: 10.1109/ISCAS45731.2020.9180808 (2020).

64. Payvand, M. \& Indiveri, G. Spike-based plasticity circuits for always-on on-line learning in neuromorphic systems. In 2019 IEEE International Symposium on Circuits and Systems (ISCAS), 1-5, DOI: 10.1109/ISCAS.2019.8702497 (2019). 


\section{Supplementary Files}

This is a list of supplementary files associated with this preprint. Click to download.

- FMsuppinfo.pdf 